\title{
Suppressor of cytokine signaling 1-modulated metalloproteinases and tissue inhibitor of metalloproteinase in pulmonary fibrosis
}

\author{
QIURUI ZHANG*, YI GUO*, RAN DONG, RANRAN DAI and MIN ZHOU \\ Department of Respiratory Medicine, Ruijin Hospital, School of Medicine, \\ Shanghai Jiao Tong University, Shanghai 200025, P.R. China
}

Received January 8, 2014; Accepted October 31, 2014

DOI: $10.3892 / \mathrm{mmr} .2015 .3810$

\begin{abstract}
An imbalance between metalloproteinases (MMPs) and tissue inhibitors of metalloproteinases (TIMPs) affects the synthesis and degradation of extracellular matrix molecules, which have an important role in the pathogenesis of pulmonary fibrosis. Lower mRNA expression levels of suppressor of cytokine signaling 1 (SOCS1) are present in fibroblasts from the lungs of pulmonary fibrosis. However, little is currently known regarding the precise role of SOCS1 has in idiopathic pulmonary fibrosis (IPF). The present study examined the expression levels of MMPs and TIMPs in A549 human epithelial lung carcinoma cells and human embryonic lung fibroblasts (HLFs) stimulated with transforming growth factor- $\beta 1$ (TGF- $\beta 1$ ) in conditions of deficiency and over-expression of SOCS1, by transfection with a lentivirus. Overexpression of SOCS1 in A549 cells and HLFs significantly inhibited the mRNA expression levels of MMP-1, MMP-2 and MMP-9 $(\mathrm{P}<0.05)$. In the A549 cells lacking SOCS1 expression, the mRNA expression levels of TIMP-1 were significantly higher compared with the control groups $(\mathrm{P}<0.01)$. Overexpression of SOCS1 partially reversed these changes. The expression levels of TIMP-1 in the HLFs with an overexpression of SOCS1 were decreased, as compared with the SOCS1-deficient HLFs following TGF- $\beta 1$ stimulation; however, this finding was not significant $(0.24 \pm 0.01$ vs. $0.53 \pm 0.02, \mathrm{P}>0.05)$. The expression levels of TIMP-2 were significantly lower in the cells overexpressing SOCS1. Conversely, the mRNA expression levels of TIMP-2 were significantly higher in the SOCS1-deficient A549 cells, as compared with all of the other groups $(\mathrm{P}<0.05)$. TIMP-4 expression levels were elevated in the A549 cells
\end{abstract}

Correspondence to: Professor Min Zhou or Dr Ranran Dai, Department of Respiratory Medicine, Ruijin Hospital, School of Medicine, Shanghai Jiao Tong University, 197 Ruijin 2nd Road, Shanghai 200025, P.R. China

E-mail: doctor_zhou_99@163.com

E-mail: drrcyy@msn.com

*Contributed equally

Key words: suppressor of cytokine signaling 1, pulmonary fibrosis, matrix metalloproteinases, tissue inhibitor of metalloproteinase and HLFs transfected with the SOCS1-deficient lentivirus. The expression levels of TIMP-4 were significantly lower in the groups overexpressing SOCS1, as compared with the other groups. These results suggest that SOCS1 may act as a suppressor of pulmonary fibrosis, by reducing the expression of MMPs and TIMPs. Therefore, SOCS1 may be a target for IPF treatment.

\section{Introduction}

Pulmonary fibrosis is a chronic, progressive and often fatal interstitial lung disorder of unknown etiology. It is characterized by an abnormal accumulation of extracellular matrix (ECM) molecules, and alternating areas of normal lung, interstitial inflammation, fibroblast migration, proliferation and fibroblastic foci. ECM remodeling is regulated by a complex interplay between epithelial cells, fibroblasts, and inflammatory cells. Abnormal wound healing in response to multiple microscopic sites of alveolar epithelial cell (AEC) injury and activation may lead to idiopathic pulmonary fibrosis (IPF). Disruption of the alveolar epithelium, altered AEC phenotypes, AEC loss and failure of epithelial repair are the distinctive features of pulmonary fibrosis. AECs are capable of promoting the migration, proliferation and activation of mesenchymal cells, along with the formation of fibroblastic/myofibroblastic foci and excessive ECM accumulation. Accumulation of ECM in the interstitial, alveolar spaces and disruption of the basement membranes occur during progression of the disease $(1,2)$. An imbalance between the synthesis and degradation of ECM molecules in the local lung microenvironment has an important role in the pathogenesis of pulmonary fibrosis (3).

Matrix metalloproteinases (MMPs) are a family of zinc-endopeptidases that are associated with fibrocyte migration throughout the basement membranes and the interstitial collagen matrix, and also in fibrotic tissue remodeling. MMPs are capable of degrading various components of connective tissue matrices, and are believed to have an important role in remodeling following parenchymal damage, leading to tissue destruction in pulmonary diseases (4). MMPs have been shown to be upregulated in pulmonary fibrosis $(5,6)$. Pivotal extracellular control of the catalytic activity of MMPs is accomplished by members of a specific family of inhibitors, the tissue inhibitors of metalloproteinases (TIMPs). There are four known members of this family (TIMP-1 to -4) that 
bind to the active site of MMPs in a 1:1 ration. TIMPs may act in the tissue environment to neutralize used proteinases, thereby preventing excessive and unwanted degradation away from the sites of metalloproteinase production (7). Following injury, the expression of MMPs is rapidly increased, and gradually declines as the wound enters the remodeling phase. This system is tightly regulated by TIMPs. Previous evidence has suggested that abnormal alteration of the MMPs/TIMPs balance under pathological conditions may lead to aberrant tissue repair, accumulation of ECM and impairment of lung function (8).

Various cytokines and chemokines are also associated with the fibrotic progress. Suppressor of cytokine signaling 1 (SOCS1) proteins are inhibitors of cytokine signaling (9). SOCS1 is usually expressed at low levels, but may be induced by various cytokines and bind to Jaks, in order to inhibit subsequent signal transduction. Shod et al (10) demonstrated that lower mRNA expression levels of SOCS1 were produced by fibroblasts from the lungs of pulmonary fibrosis. The deficiency of SOCS1 in murine fibroblasts resulted in the overproduction of collagen, which was conversely suppressed by overexpression of SOCS1. However the specific mechanisms remain unclear. The present study hypothesized that the SOCS1 gene may have a role in the modulation of ECM accumulation, by influencing the expression levels of MMP/TIMP in AECs and fibroblasts, and SOCS1 may provide a novel therapeutic strategy against pulmonary fibrosis. The expression levels of MMPs and TIMPs in AECs and fibroblasts were determined under the conditions of deficiency and overexpression of SOCS1.

\section{Materials and methods}

Construction of RNA interference targeting SOCS1. A small hairpin (sh)RNA specifically targeting human SOCS1 was produced, based on a published human SOCS1 gene sequence (http://www.ncbi.nlm.nih.gov/gene/8651). A pair of oligonucleotides synthesized by Biotelevector (Shanghai, China) were annealed to the gene sequence, and the double-stranded shRNA templates were diluted (1:50) to $200 \mathrm{nM}$, and further ligated to the lentiviral vector pP113.7, which was double digested with XhoI/HpaI (Fermentas, Thermo Fisher Scientific, Pittsburgh, PA, USA). The Pll3.7 vector lacking any insertion of oligonucleotides was used as a negative control. JM 109 Escherichia coli (E. coli) competent cells were purchased from the Cytology Center of the Chinese Academy of Science (Shanghai, China) and were transformed with the plasmids. A colony was selected and the recombinant plasmids were extracted for sequencing using a gel extraction kit.

Plasmid transformation, selection and extraction. Plasmid (1 ng) was added to $200 \mu \mathrm{l} \mathrm{JM} 109 \mathrm{E}$. coli competent cells, placed on ice for $30 \mathrm{~min}$, then at $42^{\circ} \mathrm{C}$ for $90 \mathrm{sec}$, prior to the addition of $1 \mathrm{ml} \mathrm{LB}$ medium which was then cultured for $1 \mathrm{~h}$ at $37^{\circ} \mathrm{C}$ and transferred to a $200-\mu 1$ coated plate (LB medium). For plasmid selection, a colony was selected from the plate and added to $1 \mathrm{ml}$ of the tube containing $5 \mathrm{ml} \mathrm{LB}$ medium, cultured at $37^{\circ} \mathrm{C}$ and the plasmids were subsequently extracted from the bacteria using Plasmid Maxi Preperation kit (Beyotime Institute of Biotechnology, Shanghai, China). Extraction was achieved via the addition of $250 \mu \mathrm{l}$ Solution I/RNaseA mixture, which completely suspended the bacteria. Solution II $(250 \mu \mathrm{l})$ was added to the resuspended mixture, followed by $350 \mu \mathrm{l}$ Solution III. The mixture was inverted several times to induce the formation of a white flocculent precipitate. This mixture was subsequently centrifuged at $10,000 \times \mathrm{g}$ for $10 \mathrm{~min}$, then the supernatant was transferred to a collection tube containing the Hi Bind DNA binding column, centrifuged at 10,000 x $\mathrm{g}$ for $1 \mathrm{~min}$ and the liquid was discarded. Subsequently, $500 \mu 1 \mathrm{HB}$ Buffer was added and centrifuged at $10,000 \mathrm{x} \mathrm{g}$ for a further $1 \mathrm{~min}$. The liquid was discarded and $700 \mu \mathrm{l}$ DNA wash buffer was added and centrifuged at 10,000 $\mathrm{x} g$ for $1 \mathrm{~min}$. Finally, the column was mounted on a clean $1.5 \mathrm{ml}$ tube, $30-50 \mu 1$ elution buffer was added to the column and centrifuged at $10,000 \mathrm{xg}$ for $1 \mathrm{~min}$ to elute the plasmid DNA.

Cloning of SOCS1 cDNA lentivirus. The A549 human epithelial lung carcinoma cell line was purchased from the Cytology Center of the Chinese Academy of Science. Fetal bovine serum (FBS) and Opti-MEM ${ }^{\circledR}$ were obtained from Gibco Life Technologies (Carlsbad, CA, USA). Dulbecco's modified Eagle's medium (DMEM) was purchased from Sigma-Aldrich (St Louis, MO, USA). Penicillin and streptomycin were obtained from Invitrogen Life Technologies (Carlsbad, CA, USA). Total RNA was extracted from $10^{6}$ cells using TRIzol ${ }^{\circledR}$ reagent (Invitrogen Life Technologies), and the quality and quantity of the obtained RNA was determined by spectrophotometry based on the absorbance at A260/A280. The RNA product was then reverse transcribed into cDNA using a Fermentas reverse transcription kit (Thermo Fisher Scientific). Total RNA $(1 \mu \mathrm{g})$ and $1 \mu \mathrm{l}$ oligo(dT) were added to a sterile, nuclease-free tube on ice, followed by $1 \mu 1 \mathrm{DEPC}$ water, prior to incubation at $65^{\circ} \mathrm{C}$ for $5 \mathrm{~min}$, chilling on ice and spinning down back on ice. The following components were added: $4 \mu 1$ $5 \mathrm{X}$ reaction buffer, $2 \mu 110 \mathrm{mM}$ dNTP mix, $1 \mu$ RevertAid $^{\mathrm{TM}}$ M-MuLV reverse transcriptase and $1 \mu \mathrm{l} \mathrm{RiboLock}{ }^{\mathrm{TM}}$ RNase inhibitor, and mixed gentley prior to centrifugation at $25^{\circ} \mathrm{C}$ for $5 \mathrm{~min}, 42^{\circ} \mathrm{C}$ for $60 \mathrm{~min}, 70^{\circ} \mathrm{C}$ for $5 \mathrm{~min}$ and $4^{\circ} \mathrm{C}$ for $5 \mathrm{~min}$. The SOCS1 gene was obtained by reverse transcription-polymerase chain reaction (RT-PCR). The gene was digested by Sma1 and Kpn 1 enzymes (Fermentas, Burlington, ON, Canada): Socs1-SmaI-F, 5'-TCCCCCGGGATGGTAGCACACAAC CAGGTG-3'; Socs1-KpnI-R, 5'-GGGGTACCTCAAATCTGG AAGGGGAAGG-3' (Invitrogen Life Technologies, Shanghai, China). The reagent included $8 \mu 1$ 10X TE buffer + bovine serum albumin (GaoChuang Medical Technology Co., Ltd., Shanghai, China), $50 \mu 1$ SOCS1, $1 \mu 1$ Smal, $1 \mu 1 \mathrm{Kpnl}$ and double-distilled $\mathrm{H}_{2} \mathrm{O}$ to a total of $80 \mu \mathrm{l}$, and was kept overnight at $37^{\circ} \mathrm{C}$. The target gene segment $(7.5 \mathrm{~L})$ was connected with carrier ppic9K, in $1.5 \mu \mathrm{l} 10 \mathrm{X}$ T4 buffer solution and $1 \mu \mathrm{T} 4$ ligase, at $37^{\circ} \mathrm{C}$ for $3 \mathrm{~h}$. The gene was then transfected into DH5 $\alpha$ competent cells (Cytology Center of the Chinese Academy of Science), overnight at $37^{\circ} \mathrm{C}$.

Lentiviral packaging and titration. Human embryonic kidney (HEK) 293T cells (Cytology Center of the Chinese Academy of Science) were transfected with each recombinant plasmid. For the transfection, $2 \times 10^{5}$ cells were seeded into six-well plates and an appropriate quantity of virual suspension was added, and the cells were incubated at $37^{\circ} \mathrm{C}$ for 24 hours. 
Table I. Primer sequences for the quantitative polymerase chain reaction.

\begin{tabular}{lll}
\hline Target mRNA & \multicolumn{1}{c}{ Forward primer (5'-3') } & \multicolumn{1}{c}{ Reverse primer (3'-5') } \\
\hline MMP-1 & GCACAAATCCCTTCTACCCG & ATGTCCTTGGGGTATCCGTG \\
MMP-2 & GCGATGGATACCCCTTTGAC & GTACTCCCCATCGGCGTTC \\
MMP-9 & CAGAGATGCGTGGAGAGTCG & GCAAGTCTTCCGAGTAGTTTTGG \\
TIMP-1 & TGCGGATACTTCCACAGGTC & GGGGATGGATAAACAGGGAA \\
TIMP-2 & GCACCACCCAGAAGAAGAGC & GCACAGGAGCCGTCACTTCT \\
TIMP-4 & CTCCAGTGAGAAGGTAGTTCCG & AGGGAAGAGTCAAAAGGCGT \\
\hline
\end{tabular}

MMP, matrix metalloproteinase; TIMP, tissue inhibitor of metalloproteinase.

The medium containing the virus was subsequently replaced with fresh complete medium. Following a $72 \mathrm{~h}$ incubation, the supernatants were harvested and concentrated. The viral titers were determined and calibrated in the HEK $293 \mathrm{~T}$ cell lines.

Analysis of interference and overexpression of SOCS1 gene. The supernatants of the three lentiviruses $(100 \mu \mathrm{l})$ were added to wells containing the A549 cells and human embryonic lung fibroblasts (HLFs; Cytology Center of the Chinese Academy of Science), according to the following groups: Group A, untreated cells; group B, cells transfected with the negative control lentiviral plasmid; group $\mathrm{C}$, cells transfected with the SOCS1 interfering lentiviral plasmid; and group D: cells with an overexpression of SOCS1. The supernatants were replaced with DMEM, containing 10\% FBS, after $24 \mathrm{~h}$, and the cells were incubated for a further $72 \mathrm{~h}$. Western blot analysis was used to assess the expression levels of the SOCS1 protein. Briefly, HLFs were lysed in $100 \mathrm{ml}$ lysis buffer $(150 \mathrm{mM}$ $\mathrm{NaCl}, 50$ mM Tris-Cl pH 7.4, 1 mM EDTA, 0.1\% SDS, $1.0 \%$ deoxycholatic, $1.0 \%$ TritonX-100 and $1 \mathrm{mM}$ PMSF). Protein concentrations were quantified using the bicinchoninic acid Protein Assay kit (Thermo Fisher Scientific) according to the manufacturer's instructions. The proteins were separated on $12 \%$ SDS-PAGE gels and transferred onto polyvinylidene difluoride (PVDF) membranes (EMD Millipore, Billerica, MA, USA). The PVDF membranes were incubated with rabbit polyclonal anti-human SOCS1 (1:1,000; Santa Cruz Biotechnology, Inc., Heidelberg, Germany) at $4^{\circ} \mathrm{C}$ overnight. Human $\beta$-actin (Sigma-Aldrich) was used as an internal control. The membranes were blocked with PBS containing 5\% nonfat milk for $2 \mathrm{~h}$ at room temperature. Chemiluminscent substrate ( $1 \mathrm{ml}$; Thermo Fisher Scientific) was added and the signal was detected and quantified using an enhanced chemiluminscence system (ImageQuant LAS-4000 Mini; GE Healthcare Life Sciences, Little Chalfont, UK). All samples were normalized to $\beta$-actin using Image J 1.4.3.67 software. The A549 cells and HLFs were treated using the same protocol. To determine viral titres, $1 \times 10^{5}$ cells were seeded into six-well plates and incubated for $24 \mathrm{~h}$. Lentivirus was added to the cells at concentrations ranging between $10^{-2}$ and $10^{-6}$ in a final volume of $1 \mathrm{ml}$ and incubated for $24 \mathrm{~h}$. Following incubation, the medium containing the virus was replaced with $2 \mathrm{ml}$ complete medium and incubated for $24 \mathrm{~h}$. The GFP signal was the detected (Axiovert 10; Zeiss, Oberkochen, Germany) and the number of fluorescent clones were quantified. The clones in the last two wells were assumed to be $\mathrm{X}$ and $\mathrm{Y}$ and the titre $(\mathrm{TU} / \mathrm{ml})=(\mathrm{X}+\mathrm{Y} \times 10) / 2 \times 10^{5}$.

Measurement of MMPs and TIMPs in A549 cells and HLFs. To examine the effects of the SOCS1 gene on the expression levels of MMPs/TIMPs, the A549 cells and HLFs were randomized into six groups: Cells left untreated; cells stimulated with transforming growth factor- $\beta 1$ (TGF- $\beta 1$; R\&D Systems, Minneapolis, MN, USA) (2 ng/ml) alone; cells transfected with negative lentiviral plasmid and stimulated with TGF- $\beta 1$ ( $2 \mathrm{ng} / \mathrm{ml})$; cells transfected with a lentiviral plasmid overexpressing SOCS1 and stimulated with TGF- $\beta 1$ ( $2 \mathrm{ng} / \mathrm{ml}$ ); cells transfected with a lentiviral plasmid interfering with SOCS1 expression and stimulated with TGF- $\beta 1(2 \mathrm{ng} / \mathrm{ml})$; and cells treated with TGF- $\beta 1(2 \mathrm{ng} / \mathrm{ml})$ and dexamethasone (DXM; $10^{-6} \mathrm{M}$; Biovision, San Francisco, CA, USA). All of the cells were stimulated for $\geq 72 \mathrm{~h}$. Quantitative PCR was used to detect the mRNA expression levels of MMP-1, MMP-2, MMP-9, TIMP-1, TIMP-2 and TIMP-4. The primer sequences for the PCR are listed in Table I. PCR was performed with a 7500 Fast Real-Time PCR System (Applied Biosystems Life Technologies, Foster City, CA, USA) with FastStart Universal SYBR Green (Roche Diagnostics, Basel, Switzerland) following cDNA synthesis. PCR conditions were as follows: Initial denaturation at $95^{\circ} \mathrm{C}$ for $10 \mathrm{~min}$, followed by 40 cycles of amplification at $95^{\circ} \mathrm{C}$ for $15 \mathrm{sec}$ and $60^{\circ} \mathrm{C}$ for $60 \mathrm{sec}$. Fold-change in the gene expression levels were calculated using the $2^{-\Delta \Delta \mathrm{Ct}}$ method relative to the internal reference gene GAPDH.

Statistical analysis. Statistical analyses were performed using a t-test between every two groups using SPSS 18.0 software (SPSS, Inc., Chicago, IL, USA). $\mathrm{P}<0.05$ was considered to indicate a statistically significant difference.

\section{Results}

Results of analysis of interference and overexpression of SOCS1 gene. Western blot analysis was used to assess the expression levels of the SOCS1 protein in HLF cells. The cells deficient in SOCS1, had $>50 \%$ reduction in SOCS1 protein expression levels; whereas the cells overexpressing SOCS1 had a significant upregulation of the protein. The other two groups had no significant differences in the expression 


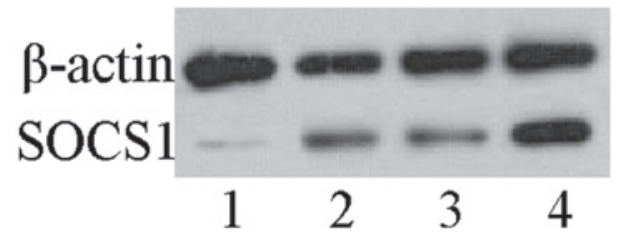

Figure 1. Western blot analysis was used to assess the expression levels of the suppressor of cytokine signaling 1 (SOCS1) protein. Lane: 1, human embryonic lung fibroblasts (HLFs) transfected with the SOCS1 interfering lentiviral plasmid; 2, untreated HLFs; 3 , HLFs transfected with the empty plasmid; 4, HLFs overexpressing SOCS1.

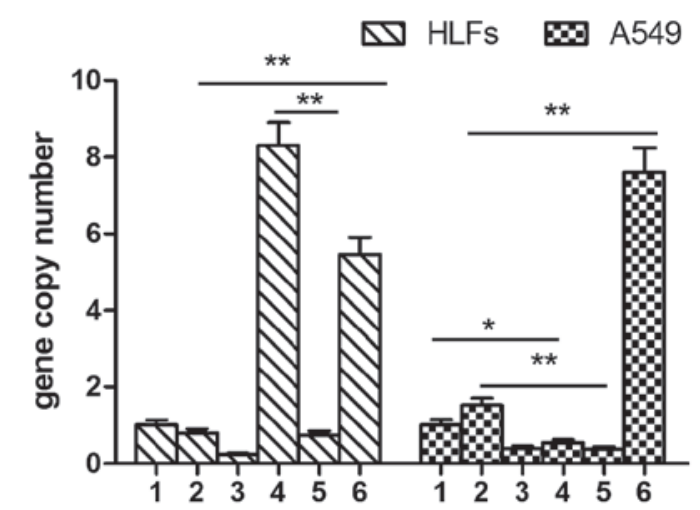

Figure 2. mRNA expression levels of matrix metalloproteinase-1 in human embryonic lung fibroblasts (HLFs) and A549 human epithelial lung carcinoma cells. 1, cells left untreated; 2 , cells stimulated with transforming growth factor (TGF)- $\beta 1$ alone; 3 , cells treated with TGF- $\beta 1$ and dexamethasone; 4 , cells transfected with the negative lentiviral plasmid and stimulated with TGF- $\beta 1 ; 5$, cells overexpressing suppressor of cytokine signaling 1 (SOCS1) and stimulated with TGF- $\beta 1 ; 6$, cells transfected with the SOCS1 interfering lentiviral plasmid and stimulated with TGF- $\beta 1$. Data are presented as the mean \pm standard deviation. ${ }^{*} \mathrm{P}<0.05,{ }^{* * *} \mathrm{P}<0.01$.

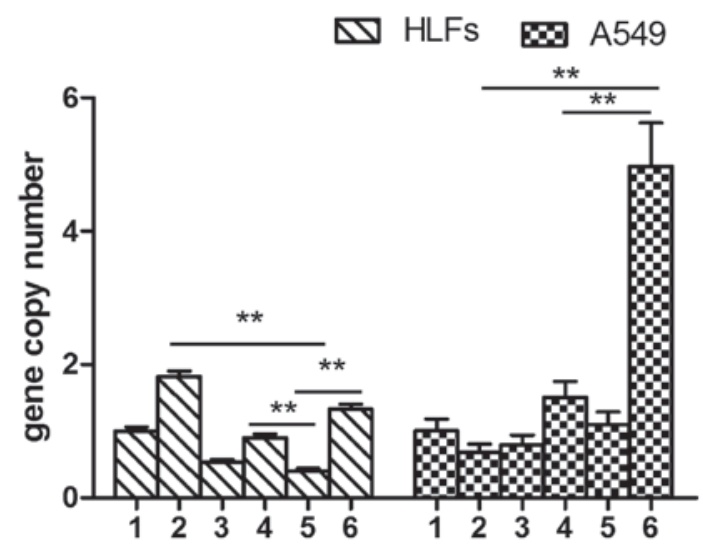

Figure 3. mRNA expression levels of matrix metalloproteinase-2 in human embryonic lung fibroblasts (HLFs) and A549 human epithelial lung carcinoma cells. 1 , cells left untreated; 2 , cells stimulated with tranforming growth factor (TGF)- $\beta 1$ alone; 3 , cells treated with TGF- $\beta 1$ and dexamethasone; 4 , cells transfected with the negative lentiviral plasmid and stimulated with TGF- $\beta 1 ; 5$, cells overexpressing suppressor of cytokine signaling 1 (SOCS1) and stimulated with TGF- $\beta 1 ; 6$, cells transfected with the SOCS1 interfering lentiviral plasmid and stimulated with TGF- $\beta 1$. Data are presented as the mean \pm standard deviation. ${ }^{*} \mathrm{P}<0.05,{ }^{* *} \mathrm{P}<0.01$.

levels of the SOCS1 protein (Fig. 1). Identical results were observed in A549 cells (data not shown).
Effects of SOCS1 gene on the mRNA expression levels of MMPs in A549 cells and HLFs. MMP-1 expression levels were slightly increased following TGF- $\beta 1$ stimulation in the A549 cells. MMP-1 expression levels in the SOCS1-deficient group were significantly higher, as compared with the A549 cells stimulated with TGF- $\beta 1(7.61 \pm 0.63$ vs. $1.52 \pm 0.19$, $\mathrm{P}<0.01)$. DXM inhibited the expression levels of MMP-1. Overexpression of SOCS1 in the A549 cells resulted in significantly lower MMP-1 expression levels, as compared with the cells stimulated with TGF- $\beta 1$ alone $(0.37 \pm 0.06$ vs. $1.52 \pm 0.19, \mathrm{P}<0.01)$. The A549 cells transfected with the negative lentiviral plasmid and stimulated with TGF- $\beta 1$ had lower MMP-1 expression levels, as compared with the untreated cells $(0.54 \pm 0.08$ vs. $1.01 \pm 0.13, \mathrm{P}<0.05)$. Conversely, TGF- $\beta 1$ did not induce the expression of MMP-1 in the HLFs. The expression levels of MMP-1 in the SOCS1-deficient HLFs was significantly higher, as compared with the HLFs treated with TGF- $\beta 1$ alone and the untreated HLFs $(5.46 \pm 0.44$ vs. $0.79 \pm 0.10, \mathrm{P}<0.01)$. The mRNA expression levels of MMP-1 in the HLFs overexpressing SOCS1 were significantly decreased, as compared with the HLFs transfected with the negative lentivirus $(0.75 \pm 0.10$ vs. $8.32 \pm 0.59, \mathrm{P}<0.01)$ (Fig. 2$)$.

The mRNA expression levels of MMP-2 in the SOCS1-deficient A549 cells were significantly higher, as compared with the A549 cells stimulated with TGF- $\beta 1$, and the A549 cells transfected with the negative lentiviral plasmid and stimulated with TGF- $\beta 1(4.97 \pm 0.65$ vs. $0.69 \pm 0.12,1.51 \pm 0.24$. $\mathrm{P}<0.01)$. The A549 cells overexpressing SOCS1, similarly to the cells treated with DXM, had lower expression levels of MMP-2, as compared with the HLF cells transfected with the negative lentivirus and the group stimulated with TGF- $\beta 1$ $(0.41 \pm 0.03$ vs. $0.90 \pm 0.06,1.82 \pm 0.08, \mathrm{P}<0.01)$. Conversely, TGF- $\beta 1$ induced MMP- 2 expression slightly more in the HLFs, as compared with the SOCS1-deficient HLFs. However, overexpression of SOCS1 reduced the expression levels of MMP-2, as compared with the SOCS1-deficient HLFs $(0.41 \pm 0.03$ vs. $1.33 \pm 0.07, \mathrm{P}<0.01$ ) (Fig. 3).

TGF- $\beta 1$ did not induce the expression of MMP-9 in either the A549 cells or the HLFs. There was no significant difference between the A549 cells transfected with the negative lentivirus and those transfected with the lentivirus interfering with SOCS1 expression $(\mathrm{P}>0.05)$. DXM had no effect on the mRNA expression levels of MMP-9 in the A549 cells. However, the mRNA expression levels of MMP-9 in the A549 cells overexpressing SOCS1 were significantly decreased, as compared with the SOCS1-deficient cells $(0.68 \pm 0.09$ vs. $3.91 \pm 0.34, \mathrm{P}<0.01)$. The expression levels of MMP-9 in the SOCS1-deficient HLFs were significantly increased, as compared with all of the other groups $(\mathrm{P}<0.01)$. The mRNA expression levels of MMP-9 in the HLFs overexpressing SOCS1 were significantly decreased, as compared with the HLFs transfected with the negative lentivirus $(4.15 \pm 0.35$ vs. $12.04 \pm 0.71, \mathrm{P}<0.01$ ) (Fig. 4).

Effects of SOCS1 gene on the mRNA expression levels of TIMPs in A549 cells and HLFs. TGF- $\beta 1$ induced TIMP-1 expression in the A549 cells; however, it had no effect on its expression in HLFs. The mRNA expression levels of TIMP-1 were markedly higher in the SOCS1-deficient A549 cells, as compared with the other groups $(\mathrm{P}<0.01)$. Overexpression of SOCS1 


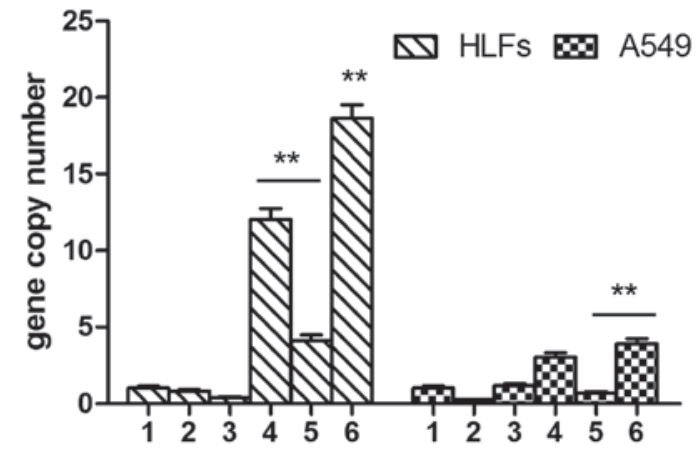

Figure 4. mRNA expression levels of matrix metalloproteinase-9 in human embryonic lung fibroblasts (HLFs) and A549 human epithelial lung carcinoma cells. 1 , cells left untreated; 2 , cells stimulated with tranforming growth factor (TGF)- $\beta 1$ alone; 3 , cells treated with TGF- $\beta 1$ and dexamethasone; cells transfected with the negative lentiviral plasmid and stimulated with TGF- $\beta 1 ; 4$, cells overexpressing suppressor of cytokine signaling 1 (SOCS1) and stimulated with TGF- $\beta 1 ; 5$, cells transfected with the SOCS1 interfering lentiviral plasmid and stimulated with TGF- $\beta 1$. Data are presented as the mean \pm standard deviation. ${ }^{*} \mathrm{P}<0.05,{ }^{* *} \mathrm{P}<0.01$.

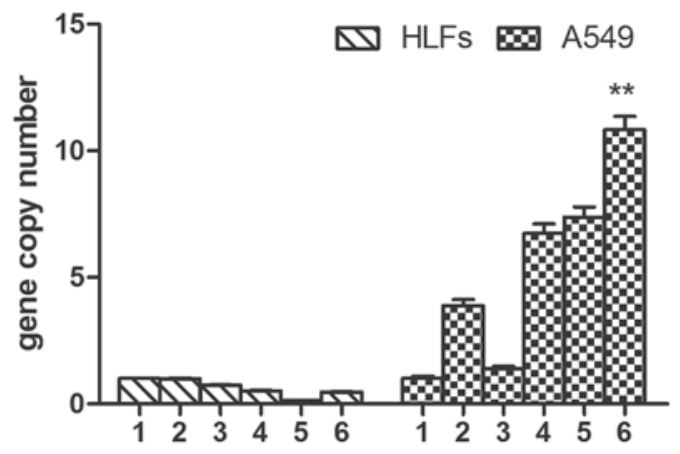

Figure 5. mRNA expression levels of tissue inhibitor of metalloproteinase-1 in human embryonic lung fibroblasts (HLFs) and A549 human epithelial lung carcinoma cells. 1, cells left untreated; 2 , cells stimulated with tranforming growth factor (TGF)- $\beta 1$ alone; 3 , cells treated with TGF- $\beta 1$ and dexamethasone; 4 , cells transfected with the negative lentiviral plasmid and stimulated with TGF- $\beta 1 ; 5$, cells overexpressing suppressor of cytokine signaling 1 (SOCS1) and stimulated with TGF- $\beta 1 ; 6$, cells transfected with the SOCS1 interfering lentiviral plasmid and stimulated with TGF- $\beta 1$. Data are presented as the mean \pm standard deviation. ${ }^{*} \mathrm{P}<0.05,{ }^{* * *} \mathrm{P}<0.01$.

partially reversed these changes. The mRNA expression levels of TIMP-1 were low in the HLFs. There were no significant changes to TIMP-1 expression following TGF- $\beta 1$ stimulation in the HLFs transfected with the negative lentivirus, or in the SOCS1-deficient HLFs $(\mathrm{P}>0.05)$. Furthermore, DXM did not affect the mRNA expression levels of TIMP-1 in the HLFs following stimulation with TGF- $\beta 1$. The expression levels of TIMP-1 in the HLFs overexpressing SOCS1 were decreased, as compared with the SOCS1-deficient HLFs following TGF- $\beta 1$ stimulation $(0.14 \pm 0.01$ vs. $0.47 \pm 0.01, \mathrm{P}>0.05)$; however these findings were not significant (Fig. 5).

The mRNA expression levels of TIMP-2 were low in the A549 cells and the expression was not elevated following TGF- $\beta 1$ stimulation. The mRNA expression levels of TIMP-2 in the SOCS1-deficient cells were slightly higher, as compared with all of the other groups $(\mathrm{P}<0.05)$. However, the expression levels of TIMP-2 were significantly decreased in the A549 cells overexpressing SOCS1, as compared with the cells transfected with

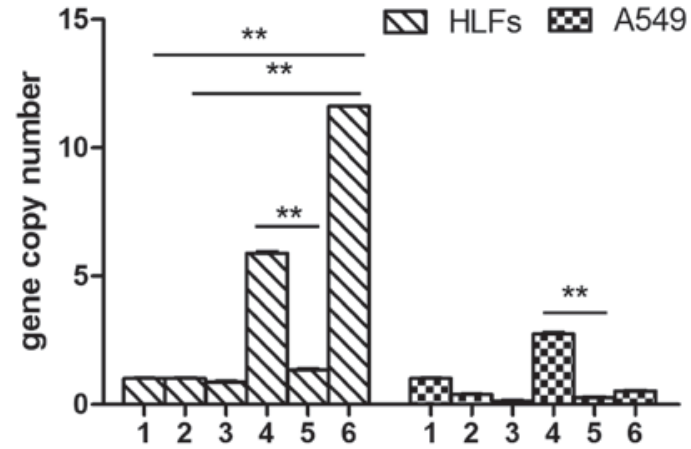

Figure 6. mRNA expression levels of tissue inhibitor of metalloproteinase-2 in human embryonic lung fibroblasts (HLFs) and A549 human epithelial lung carcinoma cells. 1, cells left untreated; 2 , cells stimulated with tranforming growth factor (TGF)- $\beta 1$ alone; 3 , cells treated with TGF- $\beta 1$ and dexamethasone; 4 , cells transfected with the negative lentiviral plasmid and stimulated with TGF- $\beta 1 ; 5$, cells overexpressing suppressor of cytokine signaling 1 (SOCS1) and stimulated with TGF- $\beta 1 ; 6$, cells transfected with the SOCS1 interfering lentiviral plasmid and stimulated with TGF- $\beta 1$. Data are presented as the mean \pm standard deviation. ${ }^{*} \mathrm{P}<0.05,{ }^{* *} \mathrm{P}<0.01$.

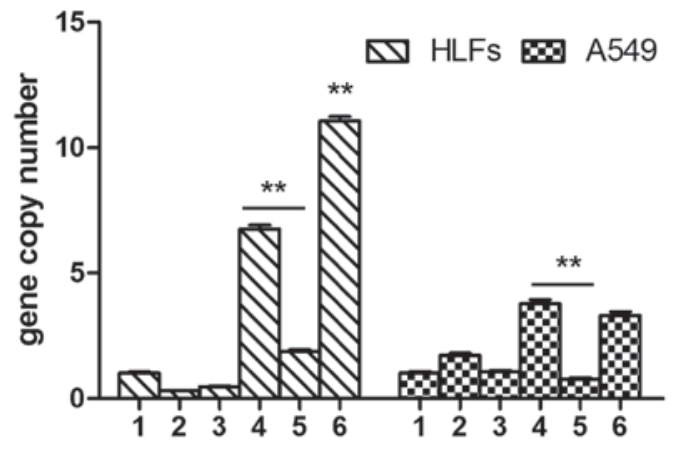

Figure 7. mRNA expression levels of tissue inhibitor of metalloproteinase-4 in human embryonic lung fibroblasts (HLFs) and A549 human epithelial lung carcinoma cells. 1, cells left untreated; 2, cells stimulated with tranforming growth factor (TGF)- $\beta 1$ alone; 3 , cells treated with TGF- $\beta 1$ and dexamethasone; 4 , cells transfected with the negative lentiviral plasmid and stimulated with TGF- $\beta 1 ; 5$, cells overexpressing suppressor of cytokine signaling 1 (SOCS1) and stimulated with TGF- $\beta 1 ; 6$, cells transfected with the SOCS1 interfering lentiviral plasmid and stimulated with TGF- $\beta 1$. Data are presented as the mean \pm standard deviation. ${ }^{*} \mathrm{P}<0.05,{ }^{* *} \mathrm{P}<0.01$

the negative lentivirus and $(0.27 \pm 0.02$ vs. $2.74 \pm 0.05 ; \mathrm{P}<0.01)$. Furthermore, TGF- $\beta 1$ could not induce TIMP- 2 expression in the HLFs. The expression levels of TIMP-2 were significantly higher in the SOCS1-deficient HLFs, as compared with the cells stimulated with TGF- $\beta 1$ ( $11.6 \pm 0.01$ vs. $1.00 \pm 0.03 ; \mathrm{P}<0.01)$ and the untreated HLFs $(11.6 \pm 0.01$ vs. $1.00 \pm 0.03 ; \mathrm{P}<0.01)$. The expression levels of TIMP-2 were significantly reduced in the HLFs overexpressing SOCS1, as compared with the HLFs transfected with the negative control lentivirus $(1.34 \pm 0.04$ vs. $5.89 \pm 0.05, \mathrm{P}<0.01$ ) (Fig. 6).

The mRNA expression levels of TIMP-4 were low in the A549 cells. The expression levels of TIMP-4 were slightly lower in the SOCS1-deficient group, as compared with the cells transfected with the negative lentivirus $(\mathrm{P}>0.05)$. The cells overexpressing SOCS1 and the cells treated with DXM had reduced expression levels of TIMP-4, as compared with all of the other A549 groups $(\mathrm{P}<0.05)$. TGF- $\beta 1$ did not induced TIMP-4 expression in the HLFs. However, TIMP-4 expression 
levels were elevated in the HLFs following transfection with the SOCS1-deficient lentivirus. The expression levels of TIMP-4 were significantly lower in the HLFs overexpressing SOCS1, as compared with the cells transfected with the negative control lentivirus and the SOCS1-deficient group (Fig. 7).

\section{Discussion}

Despite recent advances the pathophysiology of IPF remains not fully understood. However, injury to type II AECs is considered to be the key event for the initiation of the development of fibrosis (11). Progressive pulmonary fibrosis is also thought to result from dysregulated ECM control, which MMPs are believed to have important roles in. MMPs degrade various components of connective tissue matrices that are capable of remodeling following parenchymal damage, resulting in tissue destruction or the induction of repair processes in pulmonary diseases (12).

In the early stage of the disease, the expression levels of MMP-1 (interstitial collagenase) in the epitheliocytes, macrophages, fibroblasts, and myofibroblasts have been shown to be higher, as compared with in the later stages of IPF (13). MMP-1 was previously detected in the regenerated epithelial cells covering intra-alveolar fibrosis. In the present study, A549 cells and HLFs were shown to express MMP-1 at low levels. MMP-1 expression levels slightly increased following TGF- $\beta 1$ stimulation in A549 cells, whereas TGF- $\beta 1$ did not induce the expression of MMP-1 in the HLFs. However, the mRNA expression levels of MMP-1 in the SOCS1-deficient A549 cells and HLFs were significantly higher, as compared with the other groups. Furthermore, the A549 cells and HLFs overexpressing SOCS1 had significantly decreased expression levels of MMP-1. These results suggest that in different cells, the stimulator of MMP-1 may be different. However, in the A549 cells and HLFs deficient in SOCS1, which inhibits cytokine signaling, the MMP-1 expression levels were increased.

MMP-2 is a $72 \mathrm{kDa}$ gelatinase $\mathrm{A}$, that degrades collagen type IV, the major constituent of the basement membrane. MMP-2 has been shown to be upregulated in pulmonary fibrosis, and to be secreted by numerous kinds of cells in the lungs (6). The overexpression of MMP-2 is mainly associated with its ability to provoke disruption of the AEC basement membrane and enhance fibroblast invasion into the alveolar spaces $(14,15)$. The present study demonstrated that A549 cells and HLFs express MMP-2. MMP-2 mRNA expression levels in the SOCS1-deficient A549 cells were significantly higher, as compared with the other groups, and treatment with DXM inhibited MMP-2 expression. There were similar changes observed in the HLFs. These results suggest that SOCS1 may be useful for attenuating pulmonary fibrosis through the inhibition of MMP-2 production in AECs and fibroblasts.

MMP-9 is a $92 \mathrm{kDa}$ gelatinase $\mathrm{B}$, which degrades collagen type IV. Collagen type IV the major constituent of the basement membrane. Suga et al (4) demonstrated increased MMP-9 activity in IPF, particularly in those with rapid progression. In the lung parenchyma, MMP-9 was shown to be predominantly expressed in the inflammatory cells at the early stage of the disease. In later stages, type II pneumocytes and AECs at the periphery of the fibrotic foci retained MMP-9 expression, which was more prominent in the cells, suggesting that MMP-9 may have a role in the process of repair (16). The present study showed that SOCS1-deficient A549 cells and HLFs express higher levels of MMP-9, as compared with the control groups, and overexpression of SOCS1 inhibited the expression of MMP-9.

Matrix remodeling ensues with initiation of the disease with an MMPs/TIMPs imbalance favoring over activity of the enzyme to stimulate fibrogenesis (17). Previous studies have shown that upregulation of MMPs, and the imbalance with TIMPs, may lead to the release of growth factors from fibroblasts $(18,19)$. Numerous studies have detected elevated expression levels of MMP-1 and MMP-9, which occur in conjunction with alterations in the levels of their soluble inhibitors, TIMP-1 and -2 , in IPF $(3,5,6,8)$. Therefore, the present study investigated the changes to TIMP-1, TIMP-2 and TIMP-4 expression levels. TIMP-1 expression levels were low in AECs and fibroblasts. TGF- $\beta 1$ induced TIMP-1 expression in A549 cells; however, it had no effect on TIMP-1 expression in HLFs. Treatment with DXM did not alter the mRNA expression levels of TIMP-1 in the HLFs following TGF- $\beta 1$ stimulation, however DXM inhibited TIMP-1 expression in A549 cells. A lack of SOCS1 expression resulted in increased expression levels of TIMP-1, in the A549 cells, and overexpression of SOCS1 partially reversed these changes. SOCS1 had little effect on the expression of TIMP-1 in HLFs. These results suggest that SOCS1 may regulate the expression of TIMP-1 in A549 cells, but not in fibroblasts.

TIMP-2 was shown to be almost exclusively associated with fibroblast foci. Fibroblast foci are distributed throughout the lung parenchyma and indicate that fibrosis is actively ongoing (12). The present study demonstrated that TGF- $\beta 1$ could not induce TIMP-2 expression in AECs and fibroblasts. However, the expression levels were significantly higher in the SOCS1-deficient group, as compared with the HLFs stimulated with TGF- $\beta 1$. The expression levels of TIMP-2 were significantly lower in the cells overexpressing SOCS1. Conversely, the expression levels of TIMP-2 were slightly higher in the SOCS1-deficient A549 cells, as compared with all of the other groups. Therefore, it may be hypothesized that fibroblasts are the major sources of TIMP-2, and SOCS1 regulated fibrosis by influencing TIMP-2.

TIMP-4 is abundantly expressed in human cardiovascular structures, whereas all other tissues in the normal state, including lung parenchyma, are characterized by low or absent expression (20). The present study showed that the mRNA expression levels of TIMP-4 were low in the A549 cells. The expression levels were slightly higher in the SOCS1-deficient group, as compared with the cells transfected with the negative lentivirus. Overexpression of SOCS1 and treatment with DXM decreased the expression levels of TIMP-4, as compared with all of the other A549 groups. TGF- $\beta 1$ did not induce TIMP-4 expression in the HLFs. However, TIMP-4 expression levels were elevated in the HLFs transfected with the SOCS1-deficient lentivirus. The expression levels of TIMP-4 were significantly lower in the HLFs overexpressing SOCS1, as compared with the cells transfected with the negative control lentivirus and the SOCS1-deficient lentivirus.

Nakashima et al (21) previously suggested that SOCS1 expression was significantly reduced in the lung tissue from patients with IPF, as compared with that in non-IPF patients. 
Furthermore, SOCS1 expression was significantly reduced in severe fibrotic lesions, as compared with in less fibrotic lesions. SOCS1-deficient murine embryonic fibroblasts (MEF) spontaneously produced significantly higher amounts of type I collagen, as compared with the wild type MEF. Conversely, the effects of overexpression of SOCS1 were inhibition of type I collagen production from fibroblasts. In the present study, overexpression of SOCS1 regulated MMP-1, MMP-2, and MMP-9, and TIMP-1, TIMP-2 and TIMP-4 expression in AECs and fibroblasts. These results suggest that SOCS1 may act as a suppressor of pulmonary fibrosis, by reducing expression of MMPs and TIMPs. Therefore, SOCS1 may be a potential therapeutic target of IPF treatment.

\section{Acknowledgements}

The present study was supported by grants from the Science and Technology Commission of Shanghai (no. 09411951400) and the National Natural Science Foundation of China (no. 81200027).

\section{References}

1. Selman M, King TE, Pardo A, et al: Idiopathic pulmonary fibrosis: prevailing and evolving hypotheses about its pathogenesis and implications for therapy. Ann Intern Med 134: 136-151, 2001.

2. Green FH: Overview of pulmonary fibrosis. Chest 122 (6 Suppl): 334S-339S, 2002.

3. Selman M, Ruiz V, Cabrera S, et al: TIMP-1, $-2,-3$ and -4 in idiopathic pulmonary fibrosis. A prevailing nondegradative lung microenvironment? Am J Physiol Lung Cell Mol Physiol 279: L562-L574, 2000.

4. Suga M, Iyonaga K, Okamoto T, et al: Characteristic elevation of matrix metalloproteinase activity in idiopathic interstitial pneumonias. Am J Respir Crit Care Med 162: 1949-1956, 2000.

5. Nkyimbeng T, Ruppert C, Shiomi T, et al: Pivotal role of matrix metalloproteinase 13 in extracellular matrix turnover in idiopathic pulmonary fibrosis. PLoS One 8: e73279, 2013.

6. Dancer RC, Wood AM and Thickett DR: Metalloproteinases in idiopathic pulmonary fibrosis. Eur Respir J 38: 1461-1467, 2011.

7. Nagase $\mathrm{H}$ and Brew K: Designing TIMP (tissue inhibitor of metalloproteinases) variants that are selective metalloproteinase inhibitors. Biochem Soc Symp 70: 201-212, 2003.
8. Wang BL, Tu YY, Fu JF, et al: Unbalanced MMP/TIMP-1 expression during the development of experimental pulmonary fibrosis with acute paraquat poisoning. Mol Med Rep 4: 243-248, 2011.

9. Nakashima T, Yokoyama A, Onari Y, et al: Suppressor of cytokine signaling 1 inhibits pulmonary inflammation and fibrosis. J Allergy Clin Immunol 121: 1269-1276, 2008.

10. Shoda H, Yokoyama A, Nishino R, et al: Overproduction of collagen and diminished SOCS1 expression are causally linked in fibroblasts from idiopathic pulmonary fibrosis. Biochem Biophys Res Commun 353: 1004-1010, 2007.

11. Margaritopoulos GA, Giannarakis I, Siafakas NM and Antoniou KM: An update on idiopathic pulmonary fibrosis. Panminerva Med 55: 109-120, 2013.

12. Fukuda Y, Ishizaki M, Kudoh S, Kitaichi $M$ and Yamanaka $N$ : Localization of matrix metalloproteinases-1, -2 , and -9 and tissue inhibitor of metalloproteinase-2 in interstitial lung diseases. Lab Invest 78: 687-698, 1998.

13. Henry MT, McMahor K, Mackarel AJ, et al: Matrix metalloproteinases and tissue inhibiter of metalloproteinase-1 in sarcoidosis and IPF. Eur Respir J 20: 1220-1227, 2002.

14. McKeown S, Richter AG, O'Kane C, McAuley DF and Thickett DR: MMP expression and abnormal lung permeability are important determinants of outcome in IPF S. Eur Respir J 33: 77-84, 2009.

15. Kim JY, Choeng HC, Ahn C and Cho SH: Early and late changes of MMP-2 and MMP-9 in bleomycin-induced pulmonary fibrosis. Yonsei Med J 50: 68-77, 2009.

16. Ouchi H, Fujita M, Ikegame S, et al: The role of collagenases in experimental pulmonary fibrosis. Pulm Pharmacol Ther 21: 401-408, 2008.

17. Ramos C, Montaño M, García-Alvarez J, et al: Fibroblasts from idiopathic pulmonary fibrosis and normal lungs differ in growth rate, apoptosis, and tissue inhibitor of metalloproteinases expression. Am J Respir Cell Mol Biol 24: 591-598, 2001.

18. Ruiz V, Ordóñez RM, Berumen J, et al: Unbalanced collagenases/TIMP-1 expression and epithelial apoptosis in experimental lung fibrosis. Am J Physiol Lung Cell Mol Physiol 285: L1026-L1036, 2003.

19. Pardo A, Selman M and Kaminski N: Approaching the degradome in idiopathic pulmonary fibrosis. Int J Biochem Cell Biol 40: 1141-1155, 2008.

20. Koskivirta I, Rahkonen O, Mäyränpää M, et al: Tissue inhibitor of metalloproteinases 4 (TIMP4) is involved in inflammatory processes of human cardiovascular pathology. Histochem Cell Biol 126: 335-342, 2006.

21. Nakashima T, Yokoyama A, Onari Y, et al: Suppressor of cytokine signaling 1 inhibits pulmonary inflammation and fibrosis. J Allergy Clin Immunol 121: 1269-1276, 2008. 